\title{
Tritium loading study of tungsten pre-exposed to TEXTOR plasmas
}

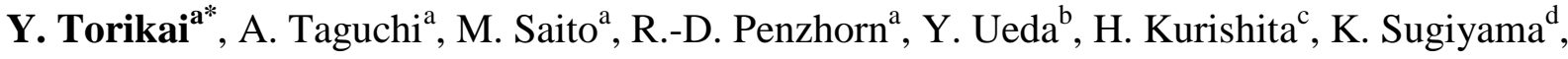 \\ V. Philipps ${ }^{\mathrm{e}}$, A. Kreter ${ }^{\mathrm{e}}$, M. Zlobinski ${ }^{\mathrm{e}}$, TEXTOR team \\ ${ }^{a}$ Hydrogen Isotope Research Center, University of Toyama, Gofuku 3190, Toyama 930-8555, Japan. \\ ${ }^{b}$ Graduate School of Engineering, Osaka University, 2-1 Yamadaoka, Suita, Osaka 565-0871, Japan. \\ ${ }^{c}$ International Research Center for Nuclear Materials Science, Institute for Materials Research (IMR), \\ Tohoku University, Oarai, Ibaraki 311-1313, Japan. \\ ${ }^{d}$ Max-Planck-Institut für Plasmaphysik, EURATOM Association, D-85748 Garching, Germany. \\ ${ }^{e}$ Institute of Energy and Climate Research - Plasma Physics, Forschungszentrum Jülich, Association \\ EURATOM-FZJ, D-52425 Jülich, Germany.
}

\begin{abstract}
The uptake of tritium at $573 \mathrm{~K}$ by polycrystalline tungsten limiter material pre-exposed to TEXTOR plasmas was investigated by the imaging plate technique (IP) and found to be mostly non-homogeneous on the plasma facing surface. Particularly high concentrations of tritium were apparent in areas attributed to carbon deposition. The surface density of tritium outside of deposition zones was essentially comparable on both sides of the examined tungsten plate. Under a stream of argon at ambient temperature tritium was predominantly released as tritiated water. While tritium is initially liberated with rates in the $(\mathrm{MBq} / \mathrm{h})$ range, after a few days the rate drops to about $100 \mathrm{~Bq} / \mathrm{h}$, decreasing even further thereafter. Under atmospheric conditions the concentration of tritium on the surface remained virtually unchanged over a rather extended period of time, i.e. more than $500 \mathrm{~d}$. Tritium in surface zones other than of "deposition" was also firmly trapped at ambient temperature.
\end{abstract}

PACS: 79.20.Rf, 52.40.Hf, 52.55.Fa, 52.25.Gj PSI-20

keywords: first-wall, TEXTOR, tritium, tungsten, limiter, imaging plate

*Corresponding author address:

Hydrogen Isotope Research Center, University of Toyama, Gofuku 3190, Toyama 9308555 , Japan.

*Corresponding author E-mail: y2torik@ctg.u-toyama.ac.jp

*Presenting author: Yuji Torikai 


\section{Introduction}

The properties of tungsten and tungsten alloys in view of their application as plasma facing components have been investigated under realistic conditions in large fusion experiments such as TEXTOR [1-3] and ASDEX [4]. In ITER tungsten is foreseen as armor material for the dome and upper part of the divertor target. Within this frame, an ITER like divertor experiment is currently in preparation at JET [5]. Tungsten is also contemplated as plasma facing component in several design studies of DEMO. High melting temperature, very high threshold for sputtering, and low hydrogen solubility may be cited among the reasons for selecting this material. However, as was shown by Nishijima et al. [6] the retention of deuterium (or tritium) in tungsten increases substantially when the material is pre-exposed to low energy helium plasmas at high temperature and decreases when instead low temperatures are selected. The annealing temperature of tungsten was also found to have a pronounced effect on the permeation rate [7]. To complete the currently available data base of tungsten as plasma facing material more detailed knowledge is needed on (i) the interdependence between tritium retention and particle fluence (approach of saturation level or linear increase?), (ii) distribution of tritium between actual surface, depositions on the surface, and bulk, and (iii) the release rate of tritium as function of temperature. This knowledge is important for a better assessment of fuel issues and tritium economy in fusion devices. Wide-ranging information on hydrogen isotope trapping in tungsten is of interest because of the associated delays in aturation, larger than expected inventories, and sudden changes in permeation rate upon variations in temperature [8].

In this study, the retention of tritium in tungsten pre-exposed in the limiter region of TEXTOR to deuterium plasmas of low-energy in the presence of small carbon impurity levels was examined by the imaging plate (IP) technique. Because the concentration of tritium in TEXTOR 
plasmas is too low for direct visualization of uptake and trap buildup by the IP technique irradiated tungsten specimens were subsequently exposed to a gaseous tritium/deuterium mixture. Long-term release rates of tritium at ambient temperature were determined using a conventional flow system.

\section{Experimental results}

A plate of polycrystalline tungsten having dimensions of $56 \times 48 \times 1 \mathrm{~mm}^{3}$ was mounted on the TEXTOR limiter at the electron diamagnetic drift side (see Fig. 1). The plasma-exposed side of the $\mathrm{W}$ plate was polished. Prior to plasma-exposure the plates were first annealed at $1273 \mathrm{~K}$ in a thermal desorption spectroscopy (TDS) chamber and additionally heated up to $633 \mathrm{~K}$ at the limiter of the TEXTOR machine in order to remove adsorbed water. During plasma exposure the temperature of the whole limiter, determined with a thermoelement placed behind the limiter, rose from initially 583 up to $623 \mathrm{~K}$. The temperature on the plate surface was measured with a pyrometer and found to increase from 713 up to $1253 \mathrm{~K}$. This large temperature difference is attributed to poor thermal contact between sample, limiter, and thermocouple and to considerable distance between sample surface and thermocouple. The tip of the limiter was $46.5 \mathrm{~cm}$ from the plasma center. The limiter was exposed in the SOL plasma to about 35 reproducible ohmic discharges with a total flattop plasma duration of $110 \mathrm{~s}$. Ions with an energy of $140 \mathrm{eV}$ were implanted with a flux of $5.4 \times 10^{22} \mathrm{D} \mathrm{m}^{-2} \mathrm{~s}^{-1}$ at a fluence of $5.9 \times 10^{24} \mathrm{D} \mathrm{m}^{-2}$. After completion of plasma irradiation the tungsten plate was removed from the limiter and cut slowly with a diamond spin saw under dry, i.e. oil-free, conditions, into smaller specimens (see left of Fig. 2) that fitted into the quartz tube used for loading. During cutting the temperature rose locally by a few $10^{\circ} \mathrm{C}$.

To get preliminary information on uptake and distribution of tritium in plasma irradiated tungsten specimens they were exposed to a tritium/deuterium mixture in a high vacuum apparatus. 
The apparatus basically consists of a turbo molecular pump (TMP), a rotary pump (RP), a quadrupole mass spectrometer (QMS), a sputter ion pump (SIP), a capacitance manometer (CM), a cold cathode gauge (CCG), a $\mathrm{ZrNi}$ tritium storage bed and a quartz vessel containing the W specimen (see Fig. 3). Getter bed and the quartz vessel are externally heated with removable ovens. Prior to loading with tritium the irradiated $\mathrm{W}$ specimens were heated at $573 \mathrm{~K}$ for $3 \mathrm{~h}$ under constant evacuation up to a final vacuum of $10^{-6} \mathrm{~Pa}$. Thereupon they were exposed to $1.2 \mathrm{kPa}$ of a deuterium/tritium mixture $\left(7.2 \% \mathrm{~T}\right.$ in $\left.\mathrm{D}^{1}\right)$ at $573 \mathrm{~K}$ for a total period of $3 \mathrm{~h}$. The selected temperature was lower than that attained in the limiter. After completion of loading, the oven of the quartz vessel was quickly removed and the specimen rapidly cooled first with water and then with liquid nitrogen. Next, the supernatant tritium/deuterium mixture was retrieved into the getter bed. Preceding specimen recovery, the loading apparatus was evacuated for 30 minutes with a sputter ion pump while keeping the quartz vessel at liquid nitrogen temperature. Finally, the specimen was removed for further investigation.

Loading conditions were such that saturation of $\mathrm{W}$ by hydrogen isotopes could be assumed. This supposition is based on calculations using solubility and diffusivity data published by Frauenfelder [9] and the one-dimensional diffusion model described in Ref. [10]. Frauenfelder's results are the best available because they were obtained at temperatures at which trapping can be neglected [11]. It may further be assumed that during loading of plasma-exposed tungsten specimens with a deuterium/tritium mixture atomic tritium permeates through the material becoming either dissolved or trapped in ion-induced and natural defects. This postulation is supported by the work of Esteban et al. [12] who showed that hydrogen is dissolved atomically in W and therefore governed by Sieverts's law. Franzen et al. [13], moreover, discussed the possibility of implanted hydrogen being captured in natural traps existing on the backside of $\mathrm{W}$ plates.

\footnotetext{
${ }^{1}$ Deuterium-tritium mixture available for experiments at Hydrogen Isotope Research Center, easily detectable when released from metals into water by liquid scintillation counting (LSC).
} 
The tritium release rate from tungsten was determined with a flow system operated at atmospheric pressure comprising a quartz tube maintained at constant temperature and a sequence water bubbler - copper oxide bed - water bubbler placed downstream [14]. The first bubbler served to trap released HTO. Other tritium containing gases, possibly released from tungsten together with HTO that exited the first bubbler were passed through a copper oxide bed maintained at $800 \mathrm{~K}$. At this temperature copper oxide will oxidize hydrogen and volatile hydrocarbons into water and carbon dioxide while retaining negligible amounts of moisture. Tritiated water generated by this procedure is trapped in the second bubbler. Aliquots from the bubblers were taken from time to time and analyzed for tritium by liquid scintillation counting.

For the determination of tritium on the surface of tungsten the imaging plate (IP) technique (Fujifilm BAS-TR) was utilized. The intensity of the photo-stimulated luminescence (PSL) was analyzed with a laser scanner (Fujifilm FLA-7000). The accessible depth of tritium in tungsten with this technique is estimated to be $<1 \mu \mathrm{m}$. Because quantitative evaluation of tritium concentrations by this technique is rather complex only relative concentrations were determined.

In a separate plasma-exposure run, two polished tungsten stripes $\left(10 \times 59 \times 5 \mathrm{~mm}^{3}\right)$, both placed between unpolished graphite stripes, were installed in the TEXTOR limiter and subjected during three days to about 30 shots per day. Only the stripe on the left of Fig. 4, here shown between two graphite stripes, was examined at HRC. After retrieval, this stripe was cut under oil, washed with water and detergent, and finally with acetone. Objective of the treatment was an attempt to remove depositions from the metal surface.

\section{Discussion}


While in the TEXTOR machine only one side of the tungsten plate had been in contact with plasmas, during subsequent loading with gaseous deuterium/tritium both sides of the specimens were equally exposed. Six specimen cut from the original $\mathrm{W}$ plate are displayed on millimeter paper on the left of Fig. 2. Imaging plates obtained from the plasma-facing side and the plasma shadowed side of these five specimens are shown in the middle of Fig. 2. Several distinct tritium concentration zones can be identified on the surfaces: (i) a high concentration zone in the range $1800-1700 \mathrm{PSL} / \mathrm{mm}^{2} / \mathrm{h}(\mathrm{A}=\mathrm{red})$, (ii) an intermediate concentration zone in the range $1100-$ $1060 \mathrm{PSL} / \mathrm{cm}^{2} / \mathrm{h}(\mathrm{B}=$ yellow$)$, and (iii) two low concentration zones with values in the range $100-$ $90 \mathrm{PSL} / \mathrm{mm}^{2} / \mathrm{h}(\mathrm{C}=$ green $)$ or $60-30 \mathrm{PSL} / \mathrm{mm}^{2} / \mathrm{h}(\mathrm{D}=$ pale blue $)$. Zones of no tritium are given by dark blue. As anticipated, zones A and B were only found on the plasma facing side. These zones are tentatively assigned to tritium trapped in thin layers of tungsten carbides deposited on the tungsten surface [15]. Carbides may actually act as diffusion barriers increasing the retention of hydrogen isotopes in the bulk [16]. Zones C and D were found on both sides of the specimens albeit with lower intensity on the plasma-shadowed side. Elevated concentrations of deuterium within $50 \mathrm{~nm}$ from the top surface of $\mathrm{W}$ having implantation-induced defects were reported by Nagata and Takahiro [17]. High trapping on radiation damaged surfaces have also been observed by Causey et al. [18] and Alimov et al. [16, 19]. In their investigations peak concentrations of deuterium were found at depths in the nanometer range from the top surface. Causey estimated that between 25 and $50 \%$ of all the hydrogen isotopes retained in $\mathrm{W}$ specimens were in the first $3-5$ $\mu \mathrm{m}$ of the plasma exposed side. It is thus not surprising to see relatively high concentrations of non-homogeneously distributed tritium on the plasma-exposed surface. This tritium can be explained by sorption in tungsten, trapping by impurities adhered to the surface or tritium held in natural traps that have withstood the annealing treatment. 
It should be noted that the limiter temperature prevailing during irradiation, i.e. $\geq 623 \mathrm{~K}$, and the temperature maintained constant during several hours of loading, i.e. $573 \mathrm{~K}$, may have reduced appreciably the number of ion-induced defects [15]. Thus, milder irradiation and loading conditions will probably give rise to an even higher retention.

Tritium on the surface of tungsten specimens appears to be rather immobile. As evident from the IP data in Table I (see also Fig. 2 right), measured at three positions of a plasma-exposed and tritium-loaded specimen stored under ambient conditions (air and $298 \mathrm{~K}$ ), there is no discernible change in tritium surface concentration at all three positions over an ageing period of 513 days.

A few additional IP results were obtained with other tungsten stripes exposed to plasmas and gaseous tritium (see Fig. 5). Of these, stripe number 1 was not exposed to plasmas and taken as reference. As apparent, this stripe also shows measurable non-homogeneously distributed tritium trapped on the surface. Stripe number 2 was subjected to perpendicular electrons and parallel ions and stripe number 3 to perpendicular ions and parallel electrons. As already observed with the W plate depicted in Fig. 2, both show high, medium and low activity zones, probably depending on kind and strength of interaction with plasmas and degree of carbon depositions. Attempts to remove deposits by washing with water, detergent, and acetone, were unsuccessful. Thus, they probably originate from plasma/W interaction and not from loosely adhered powder dispersed on the plate during cutting.

In one run a tungsten specimen having dimensions of $43 \times 15 \times 1 \mathrm{~mm}^{3}$ (see Fig. 2a position II) was introduced immediately after loading with deuterium/tritium into the flow system and the tritium release rate determined at ambient temperature using $\mathrm{Ar}$ carrier gas at flow rate of $50 \mathrm{~cm}^{3}$ $\min ^{-1}$. A comparison between the amount of tritium retained in the first and second bubbler leads to 
the conclusion that under inert carrier gas tritium is nearly completely liberated from tungsten as tritiated water. Even though the $\mathrm{HTO} / \mathrm{HT}$ ratio changes during the course of the release, it remains at all time much larger than unity. HTO was also the main tritium-containing chemical species liberated from tritium-loaded nickel [20] or stainless steel [14] during thermal desorption in a flow system. Tritium is presumably released into the gas phase via exchange with traces of water present in the carrier gas. Release rates were determined by liquid scintillation counting of aliquots taken from the two bubblers at regular intervals. Results obtained over a period adding up to almost 2600 $\mathrm{h}$ showed that tritium is initially liberated rather rapidly at rates of nearly $10^{6} \mathrm{~Bq} \mathrm{~h}^{-1}$ but that this rate drops to less than $100 \mathrm{~Bq} \mathrm{~h}^{-1}$ ] after about 4 days and continues to decrease further to even lower values (see Fig. 6). The mechanism governing the release behavior of these two tritium fractions is complex and requires further investigation. It may be speculated that the initial fast process is due to surface tritium rapidly undergoing exchange with carrier gas moisture. Thereafter the release is governed first by rapid emptying of traps and then by slow long-term bulk diffusion of dissolved tritium. That initially an important fraction of tritium is retained in ion-induced traps is validated by the total hydrogen isotopes uptake, which was found to be several orders of magnitude higher than predicted on the basis of Frauenfelder's solubility data [9]. Fig. 6 illustrates further that although the release rate of tritium from the bulk has decreased by approx a factor of 1000 the overall concentration of tritium on the surface has remained virtually unchanged over a period of more than 2500 hours. This result is well in line with IP measurements performed at positions 1, 2, and 3 of the plasma-exposed side of the W specimen shown on the right of Fig. 2 (see also Table $1)$. 


\section{Conclusions}

Scoping tests provided evidence for comparatively high levels of tritium on the surface of plasma-pre-exposed tungsten charged with a deuterium/tritium mixture. Tritium is most probably trapped in carbon depositions. IP measurements carried out periodically for a period exceeding 500 days showed that tritium in depositions as well as that sequestered in traps on the surface of pre-irradiated tungsten is not noticeably released at ambient temperature. On the other hand, there are indications that the long-term release of tritium at ambient temperature observed in a flow system arises from the tungsten bulk.

\section{Acknowledgements}

This work has been partly supported by the Ministry of Education, Culture, Sports, Science, and Technology through the IEA-TEXTOR agreement (NIFS12KETE001) and by a KAKENHI Grant-in-Aid for Scientific Research (B) 24360380.

RDP gratefully acknowledges the continuing support from the University of Toyama. 


\section{References}

[1] V. Phillips et al., J. Nucl. Mater. 241-243(1997)105-117.

[2] T. Tanabe et al., J. Nucl. Mater. 283-287(2000)1128-1133.

[3] A. Kreter et al., J. Nucl. Mater. 363-365(2007)179-183.

[4] K. Krieger el al., J. Nucl. Mater. 266-269(1999)207.

[5] J. Paméla et al., J. Nucl. Mater. 363-365 (2007) 1.

[6] D. Nishijima et al., J. Nucl. Mater. 337-339 (2005) 927-931.

[7] R.A. Anderl et al., Fusion Technol. 21 (1992) 745.

[8] G.R. Longhurst et al., Fusion Technol. 19(1991)1799.

[9] R. Frauenfelder, J. Vac. Sci. Technol. 6 (1969) 388.

[10] S. Naoe et al., Fusion Sci. Technol. 54 (2008) 515.

[11] R.A. Causey, J. Nucl. Mater. 300 (2002) 91-117.

[12] G.A. Esteban et al., J. Nucl. Mater. 295(2001)49-56.

[13] P. Franzen et al. J. Nucl. Mater. 241-243 (1997) 1082-1086.

[14] Y. Torikai et al., Fusion Sci. Technol. 48 (2005) 177.

[15] O.V. Ogorodnikova et al., J. Nucl. Mater. 313-316 (2003) 469-477.

[16] V.Kh. Alimov et al., J. Nucl. Mater. 375 (2008) 192-201.

[17] S. Nagata, K. Takahiro, J. Nucl. Mater. 283-287 (2000) 1038-1042.

[18] R.A. Causey et al., J. Nucl. Mater. 266-269 (1999) 467-471.

[19] V.Kh. Alimov et al., J. Nucl. Mater. 337-339 (2005) 619-623.

[20] M. Saito et al. Fusion Sci. Technol. 60 (2011) 1459. 
Table 1

Surface activity on plasma and tritium exposed tungsten as function of ageing

\begin{tabular}{|c|c|c|c|}
\hline $\begin{array}{c}\text { Time } \\
{[\mathrm{d}]}\end{array}$ & $\begin{array}{c}\text { Pos. 1 } \\
{\left[\mathrm{PLS} / \mathrm{mm}^{2} / \mathrm{h}\right]}\end{array}$ & $\begin{array}{c}\text { Pos. 2 } \\
{\left[\mathrm{PLS} / \mathrm{mm}^{2} / \mathrm{h}\right]}\end{array}$ & $\begin{array}{c}\text { Pos. 3 } \\
{\left[\mathrm{PLS} / \mathrm{mm}^{2} / \mathrm{h}\right]}\end{array}$ \\
\hline 0 & 1667 & 291 & 146 \\
\hline 57 & 1883 & 310 & 144 \\
\hline 168 & 1781 & 288 & 152 \\
\hline 480 & 1852 & 272 & 140 \\
\hline 513 & 1654 & 276 & 132 \\
\hline
\end{tabular}




\section{Figure captions}

Fig. 1: Tungsten plate mounted at TEXTOR limiter.

Fig. 2: Tungsten plate retrieved from the limiter after cutting into smaller segments (left picture). The picture in the middle gives IP images from the plasma facing (upper picture) and avers (lower picture) side of the tungsten plate after exposure to a gaseous deuterium/tritium mixture. Also shown is an enlarged IP image of the lower right segment from the plasma facing side of the W plate (right picture). The three positions at which the surface activity was measured as function of ageing are indicated with bold numbers. Numbers on the figure are PSL intensities in $\left(\begin{array}{lll}m m & 1 h & 1\end{array}\right)$. Broken lines identify imaged sections of the plate.

Fig. 3: Apparatus for the exposure of pre-irradiated tungsten to a mixture of deuterium/tritium.

Fig. 4: Tungsten stripes mounted at TEXTOR limiter.

Fig. 5: Picture on the left shows one unexposed tungsten stripe (1) and two tungsten stripes (2 and 3) after exposure to TEXTOR plasmas. The picture on the right depicts IP images of the same three stripes after exposure to a tritium/ deuterium mixture.

Fig. 6: Semi-logarithmic plot of the tritium release rate (०) into an argon stream at ambient temperature from pre-irradiated tungsten thermally loaded with tritium. The right ordinate reflects the tritium concentrations on the metal surface determined by IP ( $\left.{ }^{\circ}\right)$. 


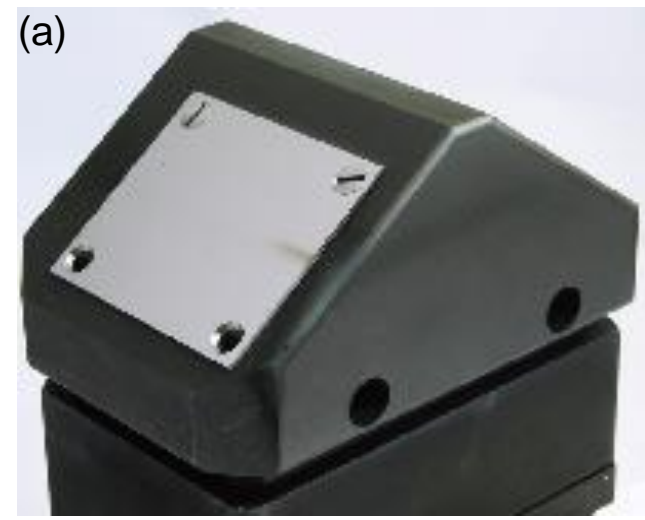

Fig. 1 
(a)

(b) Plasma facing side

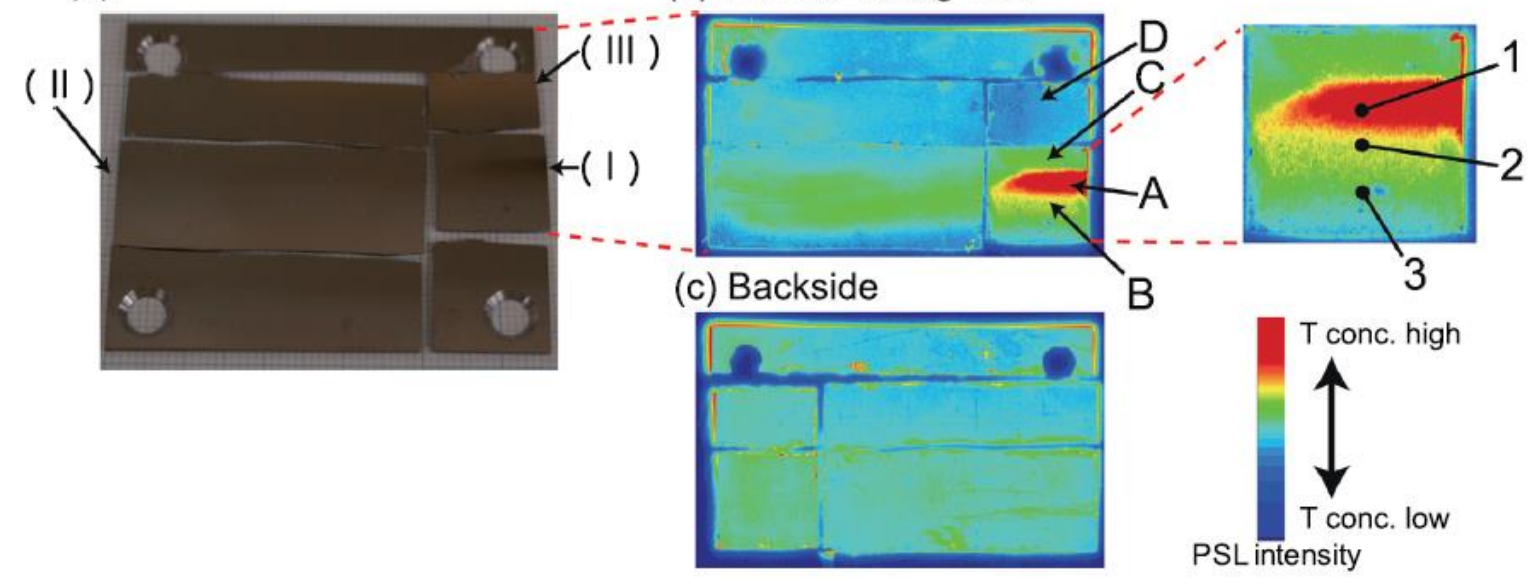

Fig. 2 


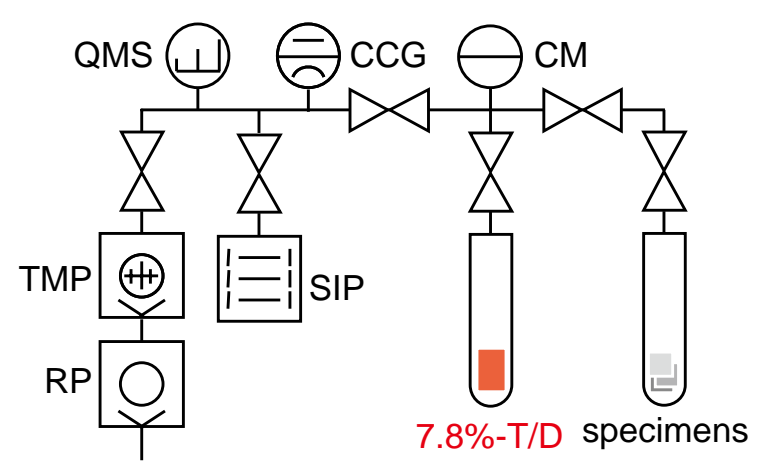

Fig. 3 


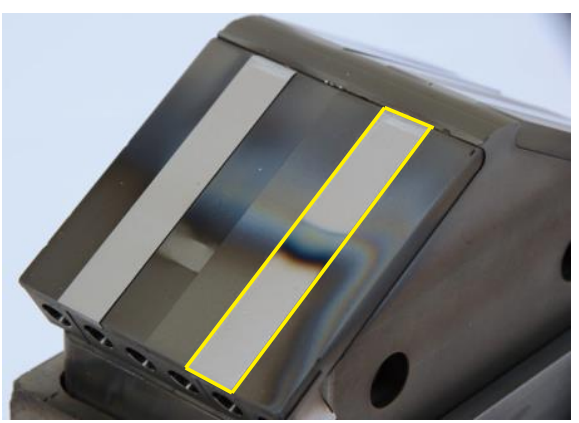

Fig. 4 


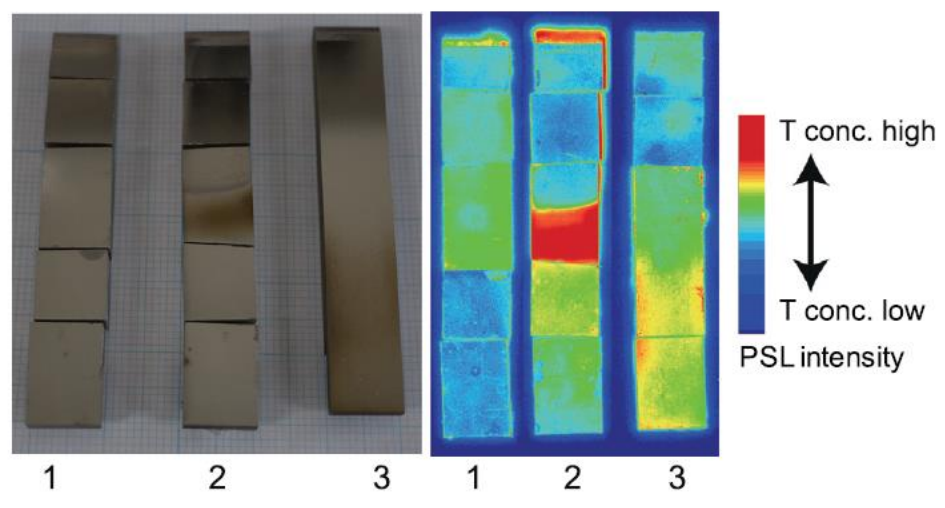

Fig. 5 


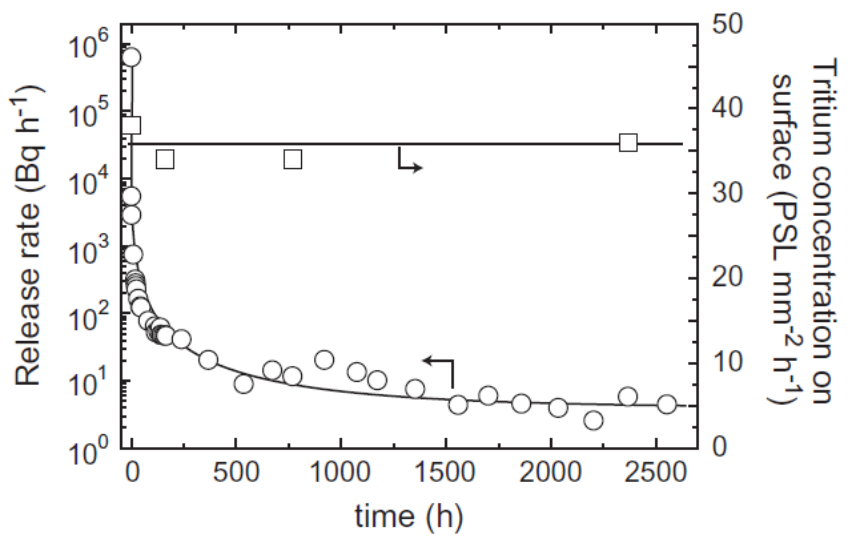

Fig. 6 